\title{
Identification of novel members of the bacterial azoreductase family in Pseudomonas aeruginosa
}

Authors: Vincenzo Crescente; Sinead M. Holland; Sapna Kashyap; Elena Polycarpou; Edith Sim; Ali $\operatorname{Ryan}^{1}$

*Faculty of Science, Engineering and Computing, Kingston University, Penhryn Road, Kingston-uponThames, UK, KT1 2EE

${ }^{1}$ Corresponding author: Dr Ali Ryan, e-mail: a.ryan@kingston.ac.uk.

Knowledge Environment: BJ Biomolecules

Running title: Characterisation of novel bacterial azoreductases

\begin{abstract}
:
Azoreductases are a family of diverse enzymes found in many pathogenic bacteria as well as distant homologues being present in eukarya. In addition to having azoreductase activity these enzymes are also suggested to have $\mathrm{NAD}(\mathrm{P}) \mathrm{H}$ quinone oxidoreductase activity which leads to a proposed role in plant pathogenesis. Azoreductases have also been suggested to play role in the mammalian pathogenesis of Pseudomonas aeruginosa. In view of the importance of $P$. aeruginosa as a pathogen, we therefore characterised recombinant enzymes following expression of a group of putative azoreductase genes from $P$. aeruginosa expressed in Escherichia coli. The enzymes include members of the "Arsenic resistance protein $\mathrm{H}^{\prime}(\mathrm{ArsH})$, "tryptophan repressor binding protein A" (WrbA), "modulator of drug activity B" (MdaB) and YieF families. The ArsH, MdaB and YieF family members all show azoreductase and $\mathrm{NAD}(\mathrm{P}) \mathrm{H}$ quinone oxidoreductase activities. In contrast, WrbA is the first enzyme to show $\mathrm{NAD}(\mathrm{P}) \mathrm{H}$ quinone oxidoreductase activity but does not reduce any of the 11 azo compounds tested under a wide range of conditions. These studies will allow further investigation of the possible role of these enzymes in the pathogenesis of $P$. aeruginosa.
\end{abstract}

Keywords: azoreductase, Pseudomonas aeruginosa, mammalian infection, modulator of drug activity B, YieF, ArsH. ${ }^{1}$

\section{Summary Statement}

Azoreductases are a family of enzymes found in many pathogenic bacteria. Members of this family are required for pathogenicity in Pseudomonas aeruginosa. Three novel classes of azoreductases were characterised, aiding in the identification of enzymes for bioremediation.

\footnotetext{
${ }^{1}$ Aqn: anthraquinone-2-sulphonate, ArsH: Arsenic resistance protein $\mathrm{H}, \mathrm{Bzq}$ : Benzoquinone, Dcb: 2,5dichlorobenzoquinone, DSF: Differential scanning fluorimetry, lbc: 2,6-dichloroquinone-4-chloromide, Jug: Juglone, Law: Lawsone, MdaB: Modulator of drug activity B, Men: Menadione NQO: NAD(P)H quinone oxidoreductase, Onq: 1,2-napthoquinone, paAzoR: $P$. aeruginosa azoreductase, Phb: Phenol blue, Plu: Plumbagin, $\mathrm{T}_{\mathrm{M}}$ : melting temperature, and WrbA: Tryptophan repressor binding protein $\mathrm{A}$.
} 


\section{Introduction}

Azoreductases are a group of flavoenzymes which have been characterised in both aerobic [1] and anaerobic bacteria [2] as well as higher organisms such as yeast [3] and mammals [4]. Although these enzymes are found ubiquitously in bacterial genomes and are constitutively expressed in vitro [5-9], their physiological role remains unclear. To date their role has been tied to their participation in the detoxification of cytotoxic quinones produced as antibacterial compounds by many plants [1]. A transcriptome study in Salmonella typhimurium has shown $>100$-fold up-regulation of an azoreductases during growth in lesions on cilantro and lettuce leaves versus in vitro growth [10]. A second study also showed significant up-regulation of Escherichia coli azoreductase during infection of radishes [6]. Studies in Pseudomonas aeruginosa using single gene deletion mutants have shown that azoreductase 1 (paAzoR1) is required for systemic infection of mice by a clinical strain of $P$. aeruginosa [11]. In addition both paAzoR1 and azoreductase 2 (paAzoR2) are required for systemic infection of mice by $P$. aeruginosa PA14 [12]. Taken as a whole, this data suggests a role for azoreductases in bacterial pathogenicity.

One of the reasons for the lack of understanding of the physiological role of this enzyme family is that in addition to azo compounds (Sup Fig 1) they reduce a broad range of substrates, including quinones [13] and nitrofurans [14]. As a result of their azoreductase activity much research has focused on the identification of azoreductases for the bioremediation of azo dye containing waste waters $[15,16]$ and engineering of these enzymes for this purpose [17]. The identification of novel families of enzymes able to reduce azo compounds would allow the exploration of a much wider variety of enzyme for the use in bioremediation and may allow dual use enzymes as some azoreductase-like enzymes can reduce heavy metals [18].

Mechanistic studies identified a common mechanism for the reduction of azo and quinone substrates $[13,19]$ and led to the proposition that azoreductases (EC: 1.7.1.6) and NAD(P)H quinone oxidoreductases (EC: 1.6.99.2) are members of a single superfamily [1]. One of the reasons these two functional classes were kept separate is the sequence diversity among the members. Proteins with sequence identities of $<10 \%$ can have the same overall fold [1] and the same enzymatic activities. This makes identification of family members via bioinformatics challenging. A recent reevaluation of the available data [1] provided evidence that the azoreductase family is significantly larger than previously thought. The superfamily is proposed to incorporate a number of previously distinct enzyme families including the Modulator of drug activity $B(\mathrm{MdaB})$, tryptophan repressor binding protein $\mathrm{A}$ (WrbA), YieF (also known as ChrR) and ArsH families. In total seven new azoreductases were predicted to be encoded by the $P$. aeruginosa PAO1 genome via an improved bioinformatics method (Table 1 [1]). In view of the challenging nature of the bioinformatics work, and the low level of sequence identity, it is important to test the hypothesis by determining whether the putative enzymes do have the activities proposed. We have therefore undertaken to characterise the azoreductases identified through bioinformatics and describe here the characterisation of 4 members of the MdaB, ArsH, WrbA and YieF, families encoded by $P$. aeruginosa. We have chosen these enzymes initially since these families occur in the genomes of many bacterial species [20-23]. 


\section{Experimental:}

All chemicals were obtained from Sigma Aldrich unless specified. NADH, NADPH and IPTG were purchased from Apollo Scientific, FMN and FAD were obtained from Tokyo Chemical Industries. Restriction enzymes were purchased from New England Biolabs.

\section{Cloning of azoreductases}

Genomic DNA was extracted from overnight cultures of $P$. aeruginosa PAO1 grown in LB at $37^{\circ} \mathrm{C}$ using a Gentra Puregene Yeast/Bacteria kit (Qiagen) and was used as a template to amplify the genes pa0949 (paWrbA), pa1204 (paYieF), pa2280 (paArsH) and pa2580 (paMdaB). PCR was performed using a Hotstar Hifidelity Taq kit (Qiagen) and the primers described in supplementary table 1. All PCR products were digested using Ndel and Sacl prior to cloning into a similarly digested pET-28b plasmid (Novagen). Ligation of the genes into pET-28b was performed using T4 DNA ligase (Promega) and pET-28b including azoreductase genes were transformed into E. coli JM109 (Promega). Transformants were selected on LB agar containing $30 \mu \mathrm{g} / \mathrm{mL}$ kanamycin. The sequences of all clones were confirmed (Eurofins MWG biotech) prior to transformation into $E$. coli BL-21 (Promega).

\section{Expression and purification of azoreductases}

All azoreductase genes were expressed as described for paAzoR1-3 [19]. Briefly, E. coli BL-21 cells were grown in LB supplemented with $1 \mathrm{M}$ Sorbitol, $2.5 \mathrm{mM}$ betaine and $30 \mu \mathrm{g} / \mathrm{mL}$ kanamycin. Cultures were grown to an $\mathrm{OD}_{600} 0.4-0.5$ and induced with $0.5 \mathrm{mM} I P T G$ for 16 hours at $18{ }^{\circ} \mathrm{C}$.

Cultures were centrifuged for 20 minutes at $6,000 \mathrm{~g}$ at $4^{\circ} \mathrm{C}$ and supernatant removed. Cells were then resuspended in lysis buffer containing complete protease inhibitors (Roche) sonication. The suspension was the centrifuged at $16,000 \mathrm{~g}$ to remove debris and the clarified supernatant applied to a Ni-NTA (Invitrogen) column. Protein was eluted via increasing imidazole concentration in the elution buffer from 0-250 mM stepwise. The exception being paYieF which required a $500 \mathrm{mM}$ imidazole wash for complete elution.

Prior to storage, all samples containing pure azoreductase were dialysed overnight against $20 \mathrm{mM}$ Tris- $\mathrm{HCl} \mathrm{pH} \mathrm{8,} 100 \mathrm{mM} \mathrm{NaCl}$ at $4^{\circ} \mathrm{C}$ in $14 \mathrm{kDa}$ cutoff dialysis tubing. Protein concentration and overall yield were determined using the following $\varepsilon_{280}$ values obtained using Protparam [24]: paWrbA $16,055 \mathrm{M}^{-1} \mathrm{~cm}^{-1}$, paYieF $-19,940 \mathrm{M}^{-1} \mathrm{~cm}^{-1}$, paArsH $-29,575 \mathrm{M}^{-1} \mathrm{~cm}^{-1}$ and paMdaB $-39,420 \mathrm{M}^{-1} \mathrm{~cm}^{-1}$. All samples were aliquoted and stored at $-80^{\circ} \mathrm{C}$ in $5 \%(\mathrm{v} / \mathrm{v})$ glycerol.

UV/VIS Absorbance scans were performed on $100 \mu \mathrm{L}$ of $1 \mathrm{mg} / \mathrm{mL}$ azoreductase in a $1 \mathrm{~mL}$ cuvette in a M200 Infite Pro (Tecan) plate reader.

\section{TLC}

Flavin cofactors were identified via the use of TLC as described previously [25]. Briefly, a mobile phase of 2:1:1 mix of n-butanol: acetic acid: water was used with a $0.2 \mathrm{~mm}$ silica gel coated plate. Pure stocks of FMN and FAD were spotted as standards for comparison. Plates were visualised under UV illumination.

\section{Differential scanning fluorimetry (DSF)}

DSF was performed via a protocol similar to that described previously [26]. Azoreductases were diluted to $64 \mu \mathrm{g} / \mathrm{mL}$ in $20 \mathrm{mM}$ Tris- $\mathrm{HCl} \mathrm{pH} \mathrm{8,100} \mathrm{mM} \mathrm{NaCl}$. The buffer contained Sypro Orange (Invitrogen) and was supplemented with either $1 \mu \mathrm{M}$ or $20 \mu \mathrm{M}$ FMN or FAD as indicated. Fluorescence measurements were made in an Mx3005p qRT-PCR instrument (Stratagene) and the temperature was increased from $25-95^{\circ} \mathrm{C}$ at $1^{\circ} \mathrm{C} / \mathrm{min}$. Data analysis was performed in Graphpad Prism with fitting to a Boltzman sigmoidal function. 


\section{Enzymatic assays}

The putative azoreductases were screened against a range of azo and quinone substrates (Sup Fig 2). Azoreductase and nitrofurazone reductase assays were performed in $100 \mu \mathrm{L}$ final volume with a reaction mixture as follows, $1.6-100 \mu \mathrm{g} / \mathrm{mL}$ azoreductase, $50 \mu \mathrm{M}$ azo/nitrofuran substrate, $500 \mu \mathrm{M}$ $\mathrm{NAD}(\mathrm{P}) \mathrm{H}, 1 \mu \mathrm{M}$ FMN/FAD, in $20 \mathrm{mM}$ Tris- $\mathrm{HCl} \mathrm{pH} 8,100 \mathrm{mM} \mathrm{NaCl}$. The concentration of enzyme was adjusted to maintain a linear rate of reaction for 1 minute to allow accurate rate determination. Reactions were monitored at the optimal wavelength for each substrate in an M200 Infinite Pro plate reader (Tecan). Rates were determined over the initial linear portion of the graph. Quinone reductase assays were performed as described previously [14] with addition of $1 \mu \mathrm{M}$ flavin to the reaction buffer.

The effect of FMN concentration on enzymatic activity was measured using Phenol Blue (Phb) as the substrate. The reaction mixture was as follows: $0.2-10 \mu \mathrm{g} / \mathrm{mL}$ azoreductase, $100 \mu \mathrm{M}$ Phb, $500 \mu \mathrm{M}$ $\mathrm{NAD}(\mathrm{P}) \mathrm{H}, 1-20 \mu \mathrm{M}$ FMN/FAD, in $20 \mathrm{mM}$ Tris- $\mathrm{HCl} \mathrm{pH} 8,100 \mathrm{mM} \mathrm{NaCl}$. A fixed concentration of each azoreductase was used for assays at all concentrations of flavin. Phb reduction was measured via the change in absorbance at $660 \mathrm{~nm}$.

Homology modelling

A homology model for paMdaB was generated using the phyre2 web server [27] in intensive mode. The paMdaB model was based upon the structures of MdaB from Yersiniae pestis (PDB: $3 R P E-59 \%$ identical) and E. coli (PDB: 2AMJ [28] - 61\% identical).

\section{Results and Discussion}

\section{Recombinant azoreductase expression}

High yields of pure recombinant paWrbA, paYieF, paArsH and paMdaB were obtained via growth and expression under the same conditions as previously used for paAzoR1-3 (Fig 1). Under these conditions the combination of E. coli BL-21 expression and His-tag purification yielded large quantities of pure protein (Fig 1) for all four azoreductases. The highest yield from a single culture was $54 \mathrm{mg}$ paYieF (paWrbA $28 \mathrm{mg}$ and paMdaB $30.2 \mathrm{mg}$ ) from $1 \mathrm{~L}$ E. coli culture.

\section{Nicotinamide cofactor selectivity}

As methyl red has previously been shown to be a good substrate for many proteins able to reduce azo compounds $[19,22]$ all four proteins were initially tested for their ability to reduce methyl red in the presence of a 5 fold molar excess of either NADH or NADPH (Fig 2). paArsH and paMdaB were able to reduce methyl red at a significantly higher rate when using NADPH compared to NADH as the nicotinamide cofactor (Fig 2C and 2D respectively).

paYieF was unable to reduce methyl red under these conditions, however when it was tested for reduction of the quinone Men it was able to reduce it in the presence of both NADH and NADPH (Fig $2 B$ ). In this case paYieF showed a significantly higher rate of reduction when using NADPH when compared to NADH. This is consistent with a previous study of paYieF which showed selectivity with NADPH [29]. Consistent with the absence of a flavin spot on the TLC plate (Sup Fig 3A), paWrbA was unable to reduce either quinones or azo compounds under these initial conditions. Instead it was necessary to add FMN to the reaction mixture to allow turnover. With the inclusion of FMN and using Men as a substrate paWrbA showed significantly higher rates of reduction of Men with NADPH as the hydride donor rather than NADH (Fig 2A). 


\section{Flavin cofactor selectivity}

Prior to characterisation of the enzyme specificity it was necessary to ascertain whether flavin was present in the recombinant azoreductases. All enzymes were expected to be flavin-dependent azoreductases however whether flavin was incorporated during expression in E. coli was uncertain. UV/Vis spectra were collected for each of the expressed azoreductases (Sup Fig 4). paWrbA lacked the characteristic flavin absorption peaks found in other azoreductases [13]. The peaks were present in all of the other proteins but were not as well defined as one would expect. In order to further investigate the incorporation of flavin measurements of absorbance at both $280 \mathrm{~nm}$ (protein) and $450 \mathrm{~nm}$ (flavin) were taken to determine the approximate protein:flavin molar ratio for the expressed proteins. One would expect a 1:1 protein:flavin molar ratio under ideal conditions [5], however the actual ratio varies from 3:1 (paMdaB) to 6:1 (paWrbA) which is consistent with the poor intensity of flavin peaks in the UV/Vis spectra. This result indicates that the flavin has either become dissociated from the expressed protein or was not sufficiently incorporated during expression. Loss or non-incorporation of flavin during recombinant expression is a frequent problem and is expected for these proteins as to obtain crystals of paWrbA and paYieF in complex with FMN it was necessary to soak preformed crystals in FMN prior to data collection [29,30]. Dissociation of flavin from the azoreductases occurs because the flavin is not covalently bound to the enzyme [19].

To confirm which flavin (FMN or FAD) was incorporated into the protein, samples were analysed via TLC (Sup Fig 3). Of the four enzymes paMdaB was the only one with an intense flavin spot via TLC (Sup Fig 3D), which suggests FAD incorporation into the enzyme. The selectivity of paMdaB for FAD and NADPH is consistent with the result from a study of $E$. coli MdaB ( $75 \%$ sequence similarity) that found the enzyme had the same cofactor preferences when reducing quinones [31]. In paWrbA and paArsH (Sup Fig $3 \mathrm{~A}$ and $\mathrm{C}$ ) a flavin spot was not present in the expressed protein and in paYieF the spot for FAD was very weak (Sup Fig 3B).

A characteristic quenching of native flavin fluorescence was observed upon titration of the recombinant azoreductases suggesting binding of the flavin to the protein. DSF was used to monitor the effect of adding extra FMN or FAD on the stability of the azoreductases. Thermostability measurements for each of the azoreductases were performed in the presence and absence of flavin and the $T_{M}$ was measured (Table 2). An increase in $T_{M}$ upon addition of flavin would indicate flavin binding to the protein. The binding of flavin within the active site will significantly stabilise the structure of the azoreductase due to the number of hydrogen bonds formed between the flavin and the protein backbone [19].

A significant thermal shift was observed upon addition of $1 \mu \mathrm{M}$ of either flavin to paMdaB however the shift was much larger with FAD than $F M N\left(16^{\circ} \mathrm{C}\right.$ with FAD compared to $5^{\circ} \mathrm{C}$ with $\left.\mathrm{FMN}\right)$. This data combined with the appearance of an FAD spot on TLC (Sup Fig $3 B$ ) suggests that FAD is the cofactor used by paMdaB. paArsH shows a small but significant thermal shift as a result of addition of $1 \mu \mathrm{M}$ FMN however no change was observed upon addition of $1 \mu M$ FAD. This suggests selective binding of FMN to the protein, which is consistent with results from ArsH in Sinorhizobium meliloti [32]. In the case of both paWrbA and paYieF no significant thermal shift was observed upon addition of either 1 or $5 \mu \mathrm{M}$ flavin and only after addition of $20 \mu \mathrm{M}$ flavin was a significant shift observed. paYieF was destabilised by addition of $20 \mu \mathrm{M} \mathrm{FMN}$, in contrast to a significant increase in thermostability $\left(>6^{\circ} \mathrm{C}\right)$ resulting from the addition of $20 \mu \mathrm{M}$ FAD. This data in combination with a weak FAD spot on TLC (Sup Fig $3 C$ ) suggests that FAD is the cofactor used by paYieF. This is inconsistent with a previous crystallographic study where the structure of paYieF was solved with FMN bound at the active site [29], however as mentioned previously the FMN was added after crystal formation and no experimental evidence was provided for the addition of FMN over FAD. paWrbA was stabilised in the presence of both FMN and FAD however the stabilisation was far greater with $\mathrm{FMN}\left(12^{\circ} \mathrm{C}\right.$ with $\mathrm{FMN}$ compared to $2^{\circ} \mathrm{C}$ with $\left.\mathrm{FAD}\right)$ suggesting $\mathrm{FMN}$ would be selectively 
incorporated into paWrbA which is consistent with FMN being highly selectively incorporated into $E$. coli WrbA [23].

In order to further analyse the flavin selectivity of the azoreductases we monitored the effect of addition of each flavin on azoreductase activity and this is covered in the NAD(P)H quinone oxidoreductase section.

\section{Azoreductase specificity profiles of the new enzymes}

paMdaB, paArsH, paWrbA and paYieF were screened against the same azo compounds which had previously been used to screen paAzoR1-3 (Sup Fig 2 [19]). Results showed that paMdaB, paArsH and paYieF were able to reduce a variety of azo compounds (Fig 3 ). As the enzymatic activity of paArsH has never been determined this data confirms its role as an azoreductase. In the cases of paMdaB and paYieF this is the first time any member of these families have been shown to reduce azo compounds.

Rates of azo bond cleavage were of the same order of magnitude as those previously seen for paAzoR1-3 [19]. Interestingly all three enzymes reduced olsalazine which was a compound which showed very little reduction by paAzoR1-3 [19]. paArsH in particular shows a propensity for azo dyes whose hydrazo-tautomers would form napthoquinones (e.g. Sudan I and olsalazine). paYieF and paMdaB do not show a particular preference for those azo dyes that form either benzo or napthoquinones in their hydrazo-tautomers.

The inclusion of the MdaB enzyme family into the azoreductase family is significant as this family is also required for systemic infection of mice in both Helicobacter pylori [20] and Francisella novocida [33] as well as $P$. aeruginosa PA14 [12]. Both paYieF and paArsH have also been shown to be required for systemic infection in mice by $P$. aeruginosa PA14 [12]. This data supports the hypothesis that azoreductases are required for bacterial pathogenicity. The reduced infectivity of azoreductase gene deletion strains may be related to a reduced ability to survive within host macrophage which has been illustrated with azoreductase gene deletion strains of a range of bacteria [34-36].

paYief is likely to be of particular use to the bioremediation industry due to its unusually high thermostability (Table 2) and the fact that members of this family are able to reduce azo bonds. A previous study showed that $E$. coli YieF is able to reduce heavy metal ions such as $\mathrm{Cr}^{6+}$ and $\mathrm{U}^{6+}$ to the less water soluble $\mathrm{Cr}^{3+}$ and $\mathrm{U}^{3+}$ [37]. As a result engineered members of the YieF family could well be used for bioremediation of both heavy metals and azo dyes. Directed evolution studies have already been performed to improve reduction of metals by E. coli YieF $[18,38]$ it is therefore possible that this could also lead to increased azo dye reduction.

In contrast paWrbA appeared to be unable to reduce any of the azo compounds we have tested under any of the conditions used in this study. The enzyme is functional due to its ability to reduce quinones (Fig 3A). The enzyme paWrbA is the most distantly related of all of the azoreductase-like enzymes thus far identified in P. aeruginosa [1]. When the overall structure of the paWrbA [30] was compared to those of classical azoreductases (e.g. paAzoR1) it was found that paWrbA showed some major differences (Fig 4). One of the key differences in the two structures is the complete truncation in paWrbA of the $\beta$-hairpin that forms the lid of the paAzoR1 active site. The residues in the $\beta$ hairpin have been show to play a crucial role in determining the substrate specificity of azoreductases [39]. In paWrbA the "lid" of the active site is formed by Trp99 (Fig 4A) which would be able to maintain some of the hydrophobic and $\pi-\pi$ stacking interactions which characterise binding of many of the substrates. The lack of the $\beta$-hairpin structure leads to a much more open active site in paWrbA compared to classical azoreductases (Fig 4C vs 4D) which would bind the azo dye much more loosely and would lead to poorer rates of reduction.

Another possible explanation for the lack of azoreduction by paWrbA is a change to an important residue in the active site of an azoreductase which is the residue equivalent to $\operatorname{Trp} 105$ in E. faecalis 
azoreductase as site directed mutagenesis has shown that substitutions at this position can completely abrogate activity [40]. This residue is an aromatic residue in most azoreductases (Tyr98 in paAzoR1 - Fig $4 \mathrm{~b}$ and Tyr96 in E. coli azoreductase) that forms $\pi$ - $\pi$ stacking interactions with FMN and also hydrogen bonds with the phosphate group of FMN. As a result this residue plays an important role in stabilising the binding of FMN to the protein. Substitution of tryptophan for small non-aromatic amino acids was shown to destabilise binding of FMN to E. faecalis azoreductase [40]. The effect of substitution to arginine (Arg80), present at this position in paWrbA (Fig 4A), was not tested however it is likely to be detrimental as arginine would be unable to form $\pi$ - $\pi$ stacking interactions and in the confirmation in the crystal structure is too far ( $4.8 \AA$ ) to form a salt bridge interaction with the phosphate group of FMN.

\section{$\mathrm{NAD}(\mathrm{P}) \mathrm{H}$ quinone oxidoreductase activity}

All four azoreductases were tested for their ability to reduce a range of quinones (Fig 5 and Sup Fig 2). For the first time paArsH was shown to reduce quinones (Fig $5 \mathrm{C}$ ). As was recently reported [41], paMdaB, paWrbA and paYieF also show quinone reductase activity. As previously shown in paAzoR13 [1] quinone reduction by these enzymes is an order of magnitude faster than reduction of azo compounds. This supports the idea that a quinone is more likely to be their physiological substrate.

paArsH as was noted for azo dye reduction, showed a preference for napthoquinone substrates (Fig $5 C$ ) with the one exception being phenol blue. Both paYieF and paMdaB also show a significant preference for napthoquinones e.g. Plu and Men (Fig 5B and D). Unlike paAzoR1-3 which all reduce Bzq at a significant rate [1] there is relatively little reduction of Bzq by any of the azoreductases tested here.

The rate of reduction of quinones by paWrbA was slower than that of the other azoreductases characterised here and is comparable to the rates of reduction previously observed for paAzoR1 [1]. These results proved to be consistent with recently published work by Green et al. [41] where paWrbA was shown to be less active than paMdaB and paYieF.

To determine whether FMN or FAD was the preferred Flavin, the reduction of Phb by the different recombinant enzymes was measured in the presence of increasing concentrations of each flavin. Phb was selected as the substrate as it is a universal substrate for the enzymes (Fig 5) and reduction was measured at $660 \mathrm{~nm}$ which would not be confounded by interference from flavin absorbance. paWrbA showed a very clear preference for FMN with no reduction observed in the presence of FAD at any of the concentrations tested (Fig 6A). This agrees with literature [30] and DSF results (table 1). Likewise the increase in rate of reduction of $\mathrm{Phb}$ by paArsH with increasing flavin concentration was much steeper with FMN than FAD (Fig 6C) agreeing with previous data suggesting that FMN is the preferred flavin. The result of addition of either FMN or FAD to paMdaB was very similar (Fig 6D) and so it was not possible to determine a preference via this method. Hence there is no reason to doubt the preference for FAD which was illustrated by the TLC data (Sup Fig 1). paYieF though is a conundrum as the enzymatic assay (Fig 6C) indicates a preference for FMN over FAD. This is inconsistent with TLC which showed a weak FAD spot (Sup Fig 1) and DSF where a small but significant $\mathrm{T}_{\mathrm{M}}$ shift was observed upon addition of $1 \mu \mathrm{M}$ FAD (table 2 ) whereas no change was observed with FMN. This data does however support a study by Agarwal et al. [29] where the crystal structure was solved in the presence of FMN although no experimental evidence was put forward for inclusion of FMN over FAD.

To help better understand the substrate specificity profiles of paYieF and paMdaB it was necessary to compare the structures of their active sites (Fig 7). With this in mind a homology model was generated for paMdaB for comparison with the the crystal structures of paAzoR1 (Fig 4B) and paYieF. The structure of the active site of paMdaB (Fig 7B) is very similar to that of paAzoR1 (Fig 4A), it is a predominantly hydrophobic pocket surrounded by aromatic residues. In paMdaB Thr87 is in a similar position to Phe60 in paAzoR1 which has previously been identified to be important for 
determining substrate specificity of azoreductases [42]. In a model of paAzor3 it was suggested that the equivalent residue (Thr60), due to its strategic position at one of the entrances to the active site, would determine the accessibility to the active site for larger substrates [19]. Both paMdaB (Fig 5D) and paAzoR3 [1] show a preference for napthoquinones which would fit with the hypothesis that the residue in this position has a gatekeeper role.

In comparison, the active site of paYieF is more open with one side dominated by the positively charged side chain of Arg100 (Fig 4B). The presence of this positive side chain might be expected to disrupt the binding of the predominantly hydrophobic substrates however rates of reduction of azo and quinone substrates are comparable to those of paMdaB (Figs 3 and 5). A possible explanation for this would be the proximity of Glu52 to the guanidinium group of Arg100 ( $\sim 3 \AA$ ) which is likely to aid in neutralising the positive charge. The proximity of these charged residues in an otherwise hydrophobic environment would facilitate the opposing ionisation states of these residues. It is worth noting that the azoreductase from E. coli (PDB $2 Z 98$ [42]) has Arg59 in a similar position to Arg100 and Arg59 was linked to the preference for interaction with specific substrate carboxyl groups.

\section{Nitrofurazone reduction}

Nitrorfurazone is a topical antibiotic which is activated by reduction of its nitro group to a hydroxylamine which is able to covalently modify DNA [14]. As well as activation by azoreductases nitrofurazone can also be activated by more specialised nitroreductases such as NfsB [42].

All of the enzymes tested were shown to reduce nitrofurazone. The rate of reduction of nitrofurazone by paYieF and paWrbA (Fig 8) are comparable to that measured previously for paAzoR1 $\left(\mathrm{V}_{\max }=9.8 \mu \mathrm{M} . \mathrm{s}^{-1} . \mathrm{mg}\right.$ of protein ${ }^{-1}$ under similar experimental conditions [14]). In contrast paArsH and paMdaB have rates that are approximately 5 and 3 fold faster respectively. This is the first evidence of nitroreductase activity of these enzymes present in $P$. aeruginosa as a previous study [43] using an indirect readout showed no activity over background during overexpression of these enzymes in cancer cell lines.

\section{Conclusions}

The work described here proves predictions [1] based on bioinformatics analysis that the azoreductase family in P. aeruginosa might be significantly larger than originally thought (Table 1 ). This was controversial due to the low sequence homology between some of the predicted enzymes. We also show that members of the widely studied bacterial MdaB and YieF families can act as azoreductases and identify an $\mathrm{NAD}(\mathrm{P}) \mathrm{H}$ quinone oxidoreductase which does not have azoreductase activity. These two activities are not inextricably linked in all enzymes. As the azoreductases which have now been identified are also known to be required for infection, this data paves the way for further investigation of their role in bacterial pathogenicity.

\section{Author Contribution}

VC, SMH and SK all collected data that was included in the manuscript. EP helped supervise the project and commented on drafts of the manuscript. AR and ES wrote the manuscript and devised as well as supervised the project.

\section{Funding:}

This work has been supported by a New Lecturer Award from the Society for Applied Microbiology. 


\section{Figure list}

\section{Fig 1: Recombinant production of pure azoreductase}

Purity of all recombinant azoreductases after IMAC chromatography determined via SDS-PAGE.

Fig 2: Nicotinamide selectivity of the azoreductase-like enzymes.

Enzymatic activities of the following azoreductase-like enzymes (A) paWrbA, (B) paYieF, (C) paArsH, (D) paMdaB. Activities were measured using the following substrates Men ( $A$ \& $B$ ) and methyl red (C $\& D)$ otherwise reactions were performed as described in materials and methods. All experiments were performed in triplicate and error bars represent \pm standard deviation. Significance was measured via a one way ANOVA ( $p<0.01)$ using Graphpad Prism.

Fig 3: Substrate specificity profiles of azoreductases reducing azo dyes.

Azoreductase activity of the following proteins (A) paYieF, (B) paArsH, (C) paMdaB. Specific activity measurements were performed as described in the materials and methods and protein concentration was adjusted to maintain a linear rate. All measurements were performed in triplicate and error bars represent \pm standard deviation.

\section{Fig 4: A comparison of the active sites of paAzoR1 and paWrbA.}

(A) The structure of the paWrbA active site. (B) The structure of the paAzoR1 active site. (C) Electrostatic surface representation of paWrbA. (D) Electrostatic surface representation of paAzoR1. In $(A)$ and $(B)$ Each monomer is coloured differently, blue and gold. FMN is shown with yellow carbon atoms and side chains are coloured according to the monomer they belong to. All images and surfaces were generated in CCP4MG [44] and were based upon PDB files paAzoR1 - 2V9C [5] and paWrbA - 1ZWL [30].

\section{Fig 5: Quinone substrate specificity profiles of azoreductases.}

Each graph represents the $\mathrm{NAD}(\mathrm{P}) \mathrm{H}$ quinone oxidoreductase activity of the following proteins $(A)$ paWrbA, (B) paYieF, (C) paArsH, (D) paMdaB. Specific activity measurements were performed as described in the materials and methods and protein concentration was adjusted to maintain a linear rate. All measurements were carried out in triplicate and error bars represent \pm standard deviation.

Fig 6: Activity assays for all azoreductases in the presence of varying concentrations of flavin.

The enzymes were as follows (A) paWrbA, (B) paYieF, (C) paArsH, (D) paMdaB. The enzyme concentration was kept constant at all concentrations of flavin, paWrbA - $10 \mu \mathrm{g} / \mathrm{mL}$, paYieF - 1 $\mu \mathrm{g} / \mathrm{mL}$, paArsH $-0.2 \mu \mathrm{g} / \mathrm{mL}$ and paMdaB $-5 \mu \mathrm{g} / \mathrm{mL}$. Phb reduction was measured by monitoring the change in absorbance at $660 \mathrm{~nm}$. Blue symbols show the effect of FMN on activity and red symbols show the effect of FAD. All measurements were performed in triplicate and error bars represent tstandard deviation.

\section{Fig 7: Comparison of the active sites of paYieF (A), and paMdaB (B).}

The structure of paYieF is based upon X-ray crystallographic data (PDB code 1X77 [29]) whereas paMdaB is based upon a homology model generated as described in the materials and methods. In both cases monomer $A$ is shown in gold while monomer $B$ is shown in light blue, while FMN (paYieF) or FAD (paMdaB) are in yellow. 


\section{Fig 8: Reduction of nitrofurazone by azoreductases}

All activity measurements were performed as described in the materials and methods section. All measurements were carried out in triplicate and error bars represent \pm standard deviation.

\begin{tabular}{|c|c|}
\hline Azoreductase & Gene code \\
\hline paAzoR1 $^{\#}$ & pa0785 \\
\hline paWrbA* $^{*}$ & pa0949 \\
\hline paYieF* $^{*}$ & pa1204 \\
\hline paNQO1 & pa1224 \\
\hline paNQO2 & $p a 1225$ \\
\hline paAzoR2 $^{\#}$ & $p a 1962$ \\
\hline paArsH $^{*}$ & pa2280 \\
\hline paMdaB* $^{*}$ & pa2580 \\
\hline paAzoR3 $^{\#}$ & pa3223 \\
\hline paNQO3 & pa4975 \\
\hline
\end{tabular}

Table 1: List of genes encoding azoreductase-like enzymes in the genome of $P$. aeruginosa PAO1

*azoreductases characterised in this manuscript. \# previously characterised azoreductases [19]. Gene numbers are as listed in the Pseudocap database [45].

\begin{tabular}{|c|c|c|c|}
\hline Azoreductase & $\mathrm{T}_{\mathrm{M}} /{ }^{\circ} \mathrm{C}$ & $\mathrm{T}_{\mathrm{M}}+\mathrm{FMN} /{ }^{\circ} \mathrm{C}$ & $\mathrm{T}_{\mathrm{M}}+\mathrm{FAD} /{ }^{\circ} \mathrm{C}$ \\
\hline paWrbA $^{\mathrm{b}}$ & $55.7 \pm 0.3$ & $67.4 \pm 0.8$ & $57.7 \pm 0.3$ \\
\hline paYieF $^{\mathrm{b}}$ & $71.1 \pm 0.6$ & $49.2 \pm 2$ & $77.3 \pm 0.5$ \\
\hline paArsH $^{\text {a }}$ & $47.3 \pm 0.1$ & $49.1 \pm 0.7$ & $47.4 \pm 0.2$ \\
\hline paMdaB $^{\mathrm{a}}$ & $47.1 \pm 0.1$ & $52.6 \pm 0.2$ & $63.5 \pm 0.1$ \\
\hline
\end{tabular}

Table 2: Binding of flavin cofactors to new azoreductases.

Changes in protein thermostability are measured in response to addition of either FMN or FAD. ${ }^{a} 1$ $\mu \mathrm{M}$ or ${ }^{\mathrm{b}} 20 \mu \mathrm{M}$ flavin. Protein was at $64 \mu \mathrm{g} / \mathrm{mL}$ in $20 \mathrm{mM}$ Tris pH $8100 \mathrm{mM} \mathrm{NaCl}$. All measurements were performed in triplicate.

\section{Sup Fig 1: Reaction scheme for the reduction of olsalazine.}

A general scheme showing the bi-bi ping pong mechanism of reduction of a typical azo compound (olsalazine) by flavin dependent azoreductases such as paAzoR1 [39].

\section{Sup Fig 2: structures for all substrates used for enzyme characterisation.}

Quinones are abbreviated as follows: Aqn: anthraquinone-2-sulphonate, Bzq: Benzoquinone, Dcb: 2,5-dichlorobenzoquinone, Ibc: 2,6-dichloroquinone-4-chloromide, Jug: Juglone, Law: Lawsone, Men: Menadione, Onq: 1,2-napthoquinone, Phb: Phenol blue and Plu: Plumbagin.

\section{Sup Fig 3: TLC plates for azoreductases.}

The TLC plates analyse flavin incorporation into the following azoreductase-like enzymes (A) paYieF, (B) paMdaB, (C) paArsH and (D) paWrbA. $20 \mu \mathrm{g}$ of enzyme and $2 \mu \mathrm{g}$ of each flavin was spotted onto the silica plate. A mobile phase of 2:1:1 N-butanol: acetic acid: water was used to achieve separation.

\section{Sup Fig 4: UV/VIS spectra of azoreductases}

The absorption spectra are for the following azoreductases (A) paYieF, (B) paMdaB, (C) paArsH, (D) paWrbA. The absorption spectra for free FMN and FAD are also shown for comparison. All spectra were collected at a protein concentration of $1 \mathrm{mg} / \mathrm{mL}$ and a path length of $1 \mathrm{~cm}$. 
References:

1 Ryan, A., Kaplan, E., Nebel, J.-C., Polycarpou, E., Crescente, V., Lowe, E., Preston, G. M. and Sim, E. (2014) Identification of NAD(P)H quinone oxidoreductase activity in azoreductases from $P$. aeruginosa: azoreductases and $\mathrm{NAD}(\mathrm{P}) \mathrm{H}$ quinone oxidoreductases belong to the same FMNdependent superfamily of enzymes. PLoS ONE. 9, e98551

2 Morrison, J. M., Wright, C. M. and John, G. H. (2012) Identification, Isolation and characterization of a novel azoreductase from Clostridium perfringens. Anaerobe. 18, 229-234

3 Liger, D., Graille, M., Zhou, C. Z., Leulliot, N., Quevillon-Cheruel, S., Blondeau, K., Janin, J. and van Tilbeurgh, H. (2004) Crystal structure and functional characterization of yeast YLR011wp, an enzyme with NAD(P)H-FMN and ferric iron reductase activities. J Biol Chem. 279, 34890-34897 4 Wu, K., Knox, R., Sun, X. Z., Joseph, P., Jaiswal, A. K., Zhang, D., Deng, P. S. K. and Chen, S. (1997) Catalytic properties of NAD(P)H:Quinone Oxidoreductase-2 (NQO2), a dihydronicotinamide riboside dependent oxidoreductase. Arch Biochem Biophys. 347, 221-228

5 Wang, C. J., Hagemeier, C., Rahman, N., Lowe, E., Noble, M., Coughtrie, M., Sim, E. and Westwood, I. (2007) Molecular cloning, characterisation and ligand-bound structure of an azoreductase from Pseudomonas aeruginosa. J Mol Biol. 373, 1213-1228

6 Landstorfer, R., Simon, S., Schober, S., Keim, D., Scherer, S. and Neuhaus, K. (2014)

Comparison of strand-specific transcriptomes of enterohemorrhagic Escherichia coli O157:H7

EDL933 (EHEC) under eleven different environmental conditions including radish sprouts and cattle feces. BMC Genomics. 15, 353

7 He, M., Li, X., Guo, L., Miller, S. J., Rensing, C. and Wang, G. (2010) Characterization and genomic analysis of chromate resistant and reducing Bacillus cereus strain SJ1. BMC Microbiol. 10, 221

8 Chen, H., Hopper, S. L. and Cerniglia, C. E. (2005) Biochemical and molecular characterization of an azoreductase from Staphylococcus aureus, a tetrameric NADPH-dependent flavoprotein. Microbiology. 151, 1433-1441

9 Liu, Z. J., Chen, H., Shaw, N., Hopper, S. L., Chen, L., Chen, S., Cerniglia, C. E. and Wang, B. C. (2007) Crystal structure of an aerobic FMN-dependent azoreductase (AzoA) from Enterococcus faecalis. Arch Biochem Biophys. 463, 68-77

10 Goudeau, D. M., Parker, C. T., Zhou, Y., Sela, S., Kroupitski, Y. and Brandl, M. T. (2013) The Salmonella transcriptome in lettuce and cilantro soft rot reveals a niche overlap with the animal host intestine. Appl Environ Microbiol. 79, 250-262

11 Rakhimova, E., Munder, A., Wiehlmann, L., Bredenbruch, F. and Tummler, B. (2008) Fitness of isogenic colony morphology variants of Pseudomonas aeruginosa in murine airway infection. PLoS One. 3, e1685

12 Skurnik, D., Roux, D., Aschard, H., Cattoir, V., Yoder-Himes, D., Lory, S. and Pier, G. B. (2013) A comprehensive analysis of In Vitro and In Vivo genetic fitness of Pseudomonas aeruginosa using high-throughput sequencing of transposon libraries. PLoS Pathog. 9, e1003582

13 Ryan, A., Wang, C. J., Laurieri, N., Westwood, I. and Sim, E. (2010) Reaction mechanism of azoreductases suggests convergent evolution with quinone oxidoreductases. Protein Cell. 1, 780-790 14 Ryan, A., Kaplan, E., Laurieri, N., Lowe, E. and Sim, E. (2011) Activation of nitrofurazone by azoreductases: multiple activities in one enzyme. Sci Rep. 1, DOI:10.1038/srep00063

15 Mendes, S., Farinha, A., Ramos, C. G., Leitao, J. H., Viegas, C. A. and Martins, L. O. (2011) Synergistic action of azoreductase and laccase leads to maximal decolourization and detoxification of model dye-containing wastewaters. Bioresour Technol. 102, 9852-9859

16 Lang, W., Sirisansaneeyakul, S., Ngiwsara, L., Mendes, S., Martins, L. O., Okuyama, M. and Kimura, A. (2013) Characterization of a new oxygen-insensitive azoreductase from Brevibacillus laterosporus TISTR1911: toward dye decolorization using a packed-bed metal affinity reactor. Bioresour Technol. 150, 298-306

17 Brissos, V., Goncalves, N., Melo, E. P. and Martins, L. O. (2014) Improving kinetic or thermodynamic stability of an azoreductase by directed evolution. PLoS One. 9, e87209 
18 Barak, Y., Ackerley, D. F., Dodge, C. J., Banwari, L., Alex, C., Francis, A. J. and Matin, A. (2006) Analysis of novel soluble chromate and uranyl reductases and generation of an improved enzyme by directed evolution. Appl Environ Microbiol. 72, 7074-7082

19 Ryan, A., Laurieri, N., Westwood, I., Wang, C. J., Lowe, E. and Sim, E. (2010) A novel mechanism for azoreduction. J Mol Biol. 400, 24-37

20 Wang, G. and Maier, R. J. (2004) An NADPH quinone reductase of Helicobacter pylori plays an important role in oxidative stress resistance and host colonization. Infect Immun. 72, 1391-1396 21 Gonzalez, C. F., Ackerley, D. F., Lynch, S. V. and Matin, A. (2005) ChrR, a soluble quinone reductase of Pseudomonas putida that defends against $\mathrm{H}_{2} \mathrm{O}_{2}$. J Biol Chem. 280, 22590-22595

22 Vorontsov, II, Minasov, G., Brunzelle, J. S., Shuvalova, L., Kiryukhina, O., Collart, F. R. and Anderson, W. F. (2007) Crystal structure of an apo form of Shigella flexneri ArsH protein with an NADPH-dependent FMN reductase activity. Protein science 16, 2483-2490

23 Kishko, I., Carey, J., Reha, D., Brynda, J., Winkler, R., Harish, B., Guerra, R., Ettrichova, O., Kukacka, Z., Sheryemyetyeva, O., Novak, P., Kuty, M., Kuta Smatanova, I., Ettrich, R. and Lapkouski, M. (2013) 1.2 Å resolution crystal structure of Escherichia coli WrbA holoprotein. Acta Crystallogr D Biol Crystallogr. 69, 1748-1757

24 Gasteiger, E., Hoogland, C., Gattiker, A., Duvaud, A., Wilkins, M., Appel, R. D. and Bairoch, A. (2005) Protein Identification and Analysis Tools on the ExPASy Server. In The Proteomics Protocols Handbook (Walker, J. M., ed.). pp. 571-607, Humana Press, Totowa

25 Liu, J., Krulwich, T. A. and Hicks, D. B. (2008) Purification of two putative type II NADH dehydrogenases with different substrate specificities from alkaliphilic Bacillus pseudofirmus OF4. Biochim Biophys Acta. 1777, 453-461

26 Niesen, F. H., Berglund, H. and Vedadi, M. (2007) The use of differential scanning fluorimetry to detect ligand interactions that promote protein stability. Nat Protoc. 2, 2212-2221

27 Kelley, L. A., Mezulis, S., Yates, C. M., Wass, M. N. and Sternberg, M. J. (2015) The Phyre2 web portal for protein modeling, prediction and analysis. Nat Protoc. 10, 845-858

28 Adams, M. A. and Jia, Z. (2006) Modulator of drug activity B from Escherichia coli: crystal structure of a prokaryotic homologue of DT-diaphorase. J Mol Biol. 359, 455-465

29 Agarwal, R., Bonanno, J. B., Burley, S. K. and Swaminathan, S. (2006) Structure determination of an FMN reductase from Pseudomonas aeruginosa PA01 using sulfur anomalous signal. Acta Crystallogr D. 62, 383-391

30 Gorman, J. and Shapiro, L. (2005) Crystal structures of the tryptophan repressor binding protein WrbA and complexes with flavin mononucleotide. Protein Sci. 14, 3004-3012

31 Hayashi, M., Ohzeki, H., Shimada, H. and Unemoto, T. (1996) NADPH-specific quinone reductase is induced by 2-methylene-4-butyrolactone in Escherichia coli. Biochim Biophys Acta. 1273, $165-170$

32 Ye, J., Yang, H. C., Rosen, B. P. and Bhattacharjee, H. (2007) Crystal structure of the flavoprotein ArsH from Sinorhizobium meliloti. FEBS Lett. 581, 3996-4000

33 Kraemer, P. S., Mitchell, A., Pelletier, M. R., Gallagher, L. A., Wasnick, M., Rohmer, L., Brittnacher, M. J., Manoil, C., Skerett, S. J. and Salama, N. R. (2009) Genome-wide screen in Francisella novicida for genes required for pulmonary and systemic infection in mice. Infect Immun. 77, 232-244

34 Sabbagh, S. C., Lepage, C., McClelland, M. and Daigle, F. (2012) Selection of Salmonella enterica serovar Typhi genes involved during interaction with human macrophages by screening of a transposon mutant library. PLoS One. 7, e36643

35 Asare, R., Akimana, C., Jones, S. and Abu Kwaik, Y. (2010) Molecular bases of proliferation of Francisella tularensis in arthropod vectors. Environ Microbiol. 12, 2587-2612

36 Green, L. (2012) in vitro and in vivo characterisation of $P$. aeruginosa oxidoreductase enzymes in pathogenesis and therapy. In School of Biological Sciences ed.)^eds.). p. 205, Victoria University of Wellington, Wellington, NZ 
37 Ackerley, D. F., Gonzalez, C. F., Park, C. H., Blake, R., 2nd, Keyhan, M. and Matin, A. (2004) Chromate-reducing properties of soluble flavoproteins from Pseudomonas putida and Escherichia coli. Appl Environ Microbiol. 70, 873-882

38 Barak, Y., Nov, Y., Ackerley, D. F. and Matin, A. (2008) Enzyme improvement in the absence of structural knowledge: a novel statistical approach. ISME J. 2, 171-179

39 Wang, C. J., Laurieri, N., Abuhammad, A., Lowe, E., Westwood, I., Ryan, A. and Sim, E. (2010)

Role of Tyrosine 131 in the active site of paAzoR1, an azoreductase with specificity for the inflammatory bowel disease pro-drug balsalazide. Acta Crystallogr F. 66, 2-7

40 Chen, H., Xu, H., Kweon, O., Chen, S. and Cerniglia, C. E. (2008) Functional role of Trp-105 of Enterococcus faecalis azoreductase (AzoA) as resolved by structural and mutational analysis.

Microbiology. 154, 2659-2667

41 Green, L. K., La Flamme, A. C. and Ackerley, D. F. (2014) Pseudomonas aeruginosa MdaB and WrbA are water-soluble two-electron quinone oxidoreductases with the potential to defend against oxidative stress. J Microbiol. 52, 771-777

42 Race, P. R., Lovering, A. L., Green, R. M., Ossor, A., White, S. A., Searle, P. F., Wrighton, C. J. and Hyde, E. I. (2005) Structural and mechanistic studies of Escherichia coli nitroreductase with the antibiotic nitrofurazone. Reversed binding orientations in different redox states of the enzyme. J Biol Chem. 280, 13256-13264

43 Green, L. K., Syddall, S. P., Carlin, K. M., Bell, G. D., Guise, C. P., Mowday, A. M., Hay, M. P., Smaill, J. B., Patterson, A. V. and Ackerley, D. F. (2013) Pseudomonas aeruginosa NfsB and nitro-CBI$\mathrm{DEl}-$-a promising enzyme/prodrug combination for gene directed enzyme prodrug therapy. Mol Cancer. 12, 58

44 McNicholas, S., Potterton, E., Wilson, K. S. and Noble, M. E. M. (2011) Presenting your structures: the CCP4mg molecular-graphics software. Acta Crystallogr D. 67, 386-394

45 Winsor, G. L., Lam, D. K., Fleming, L., Lo, R., Whiteside, M. D., Yu, N. Y., Hancock, R. E. and Brinkman, F. S. (2011) Pseudomonas Genome Database: improved comparative analysis and population genomics capability for Pseudomonas genomes. Nucleic Acids Res. 39, D596-600

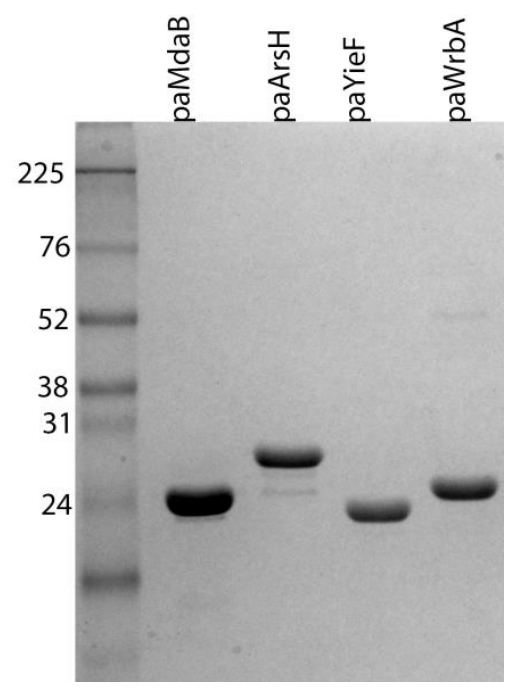

Figure 1 

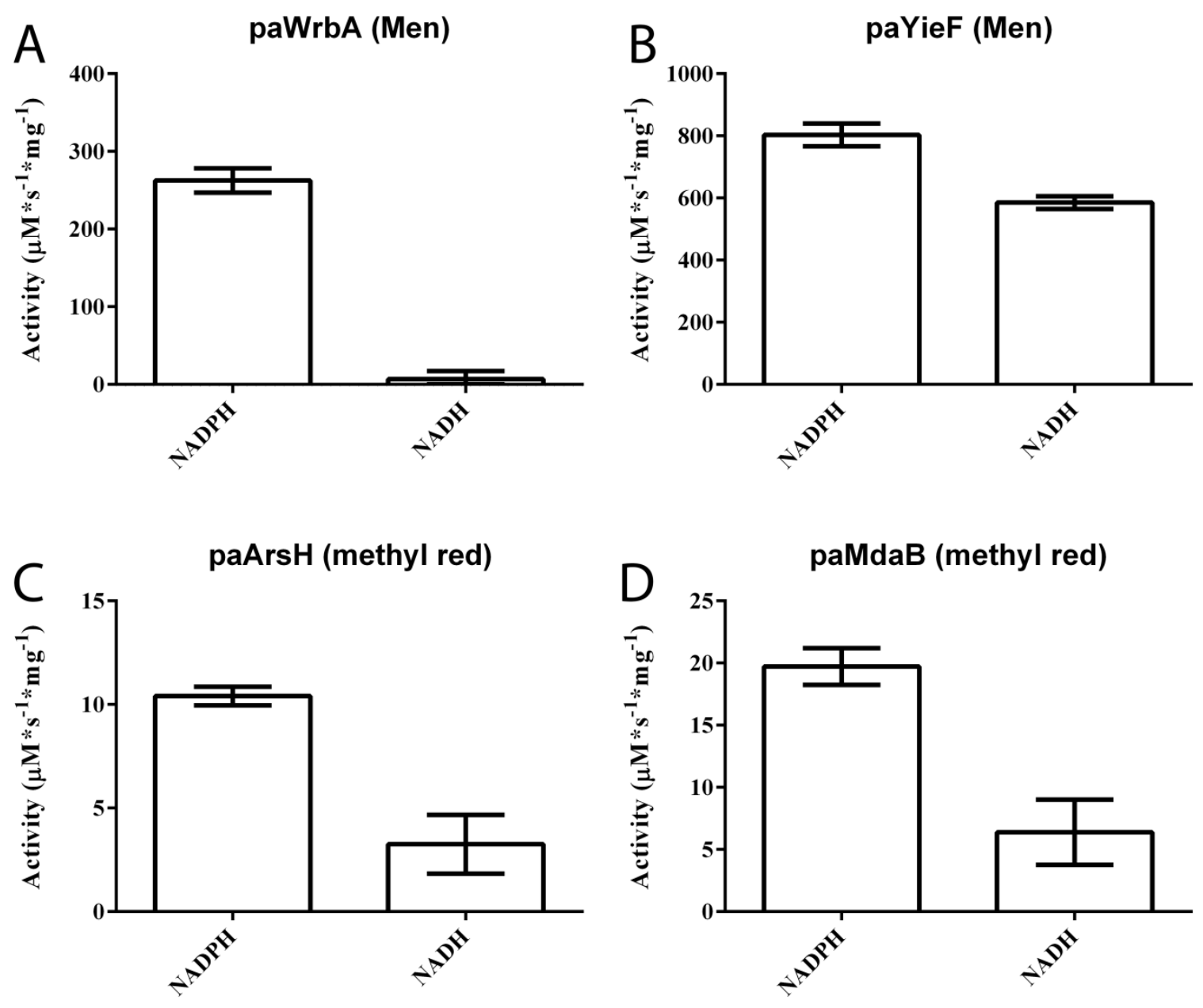

Figure 2 

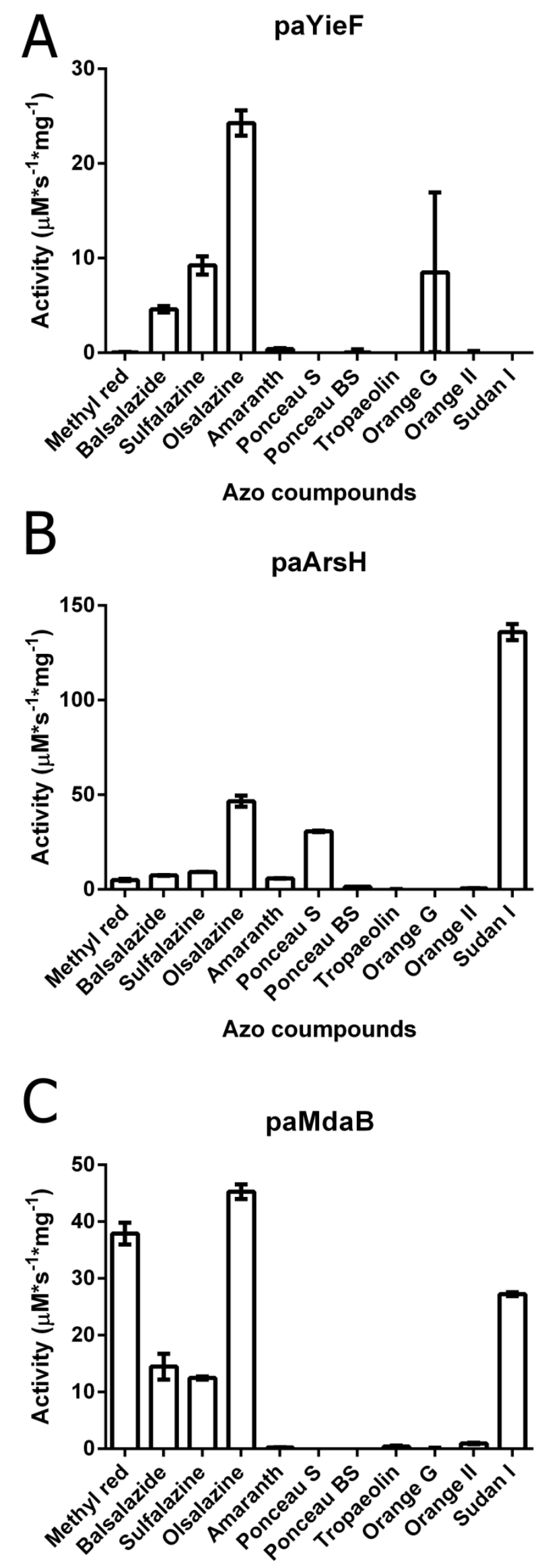

Azo coumpounds

Figure 3 


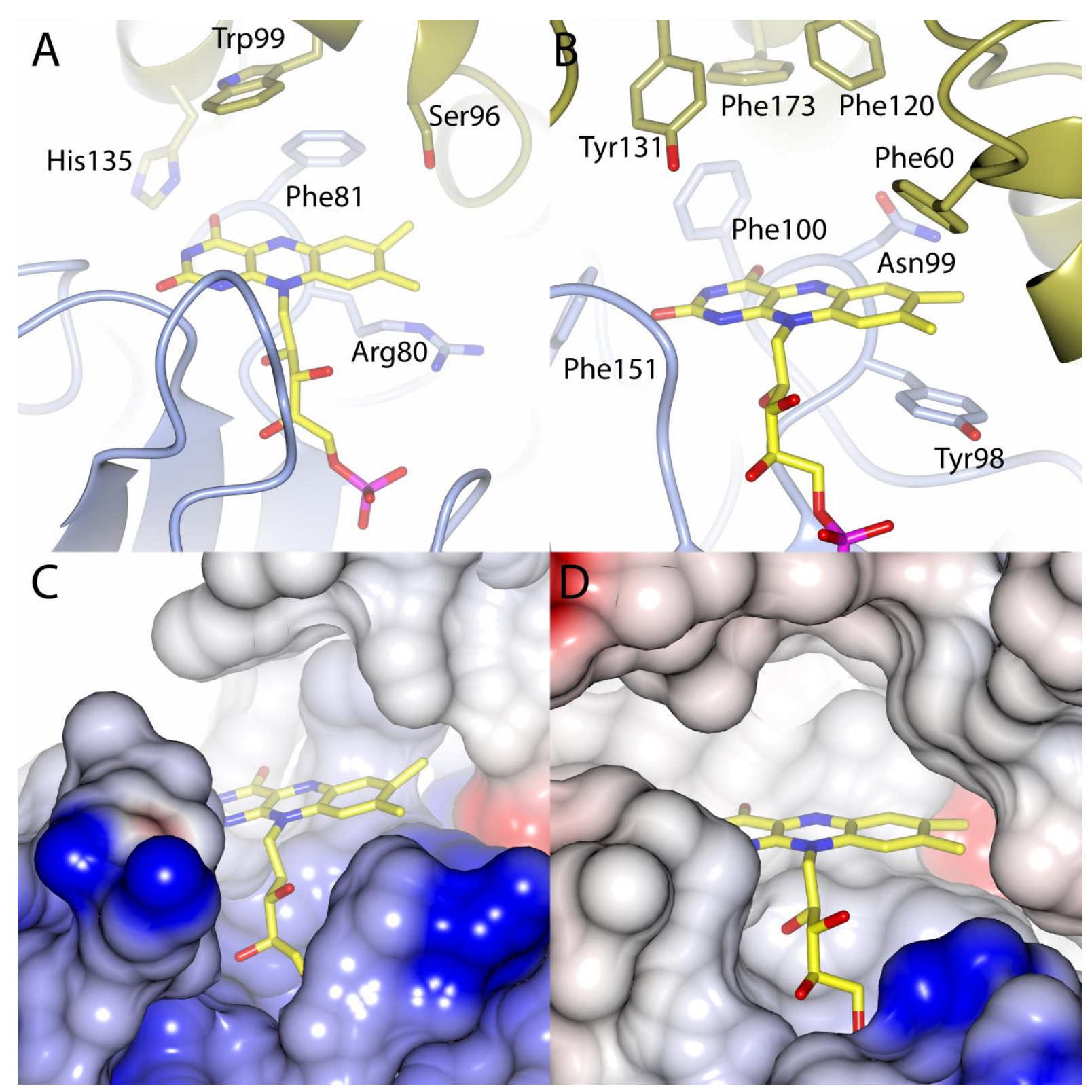

Figure 4 

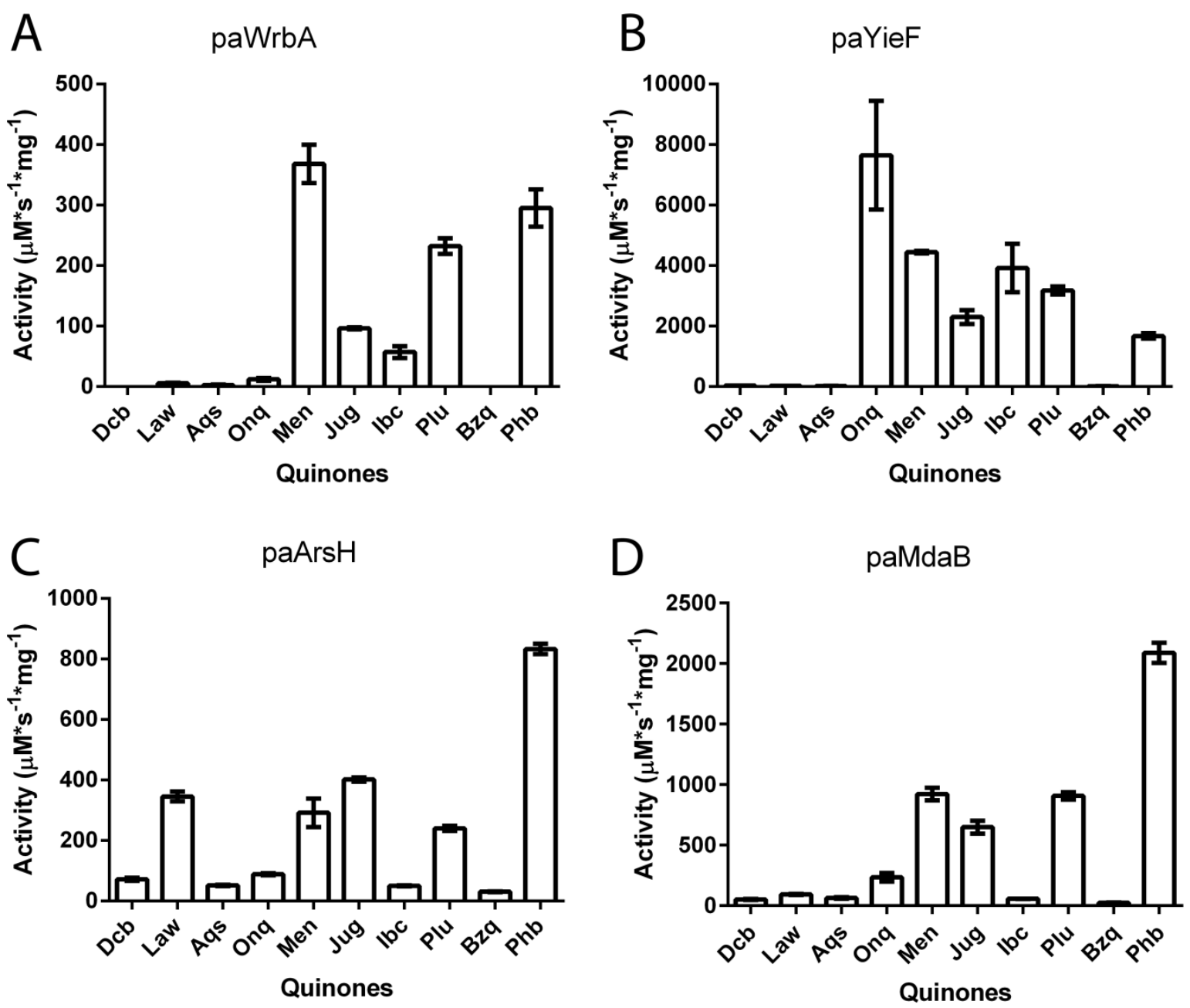

Figure 5 

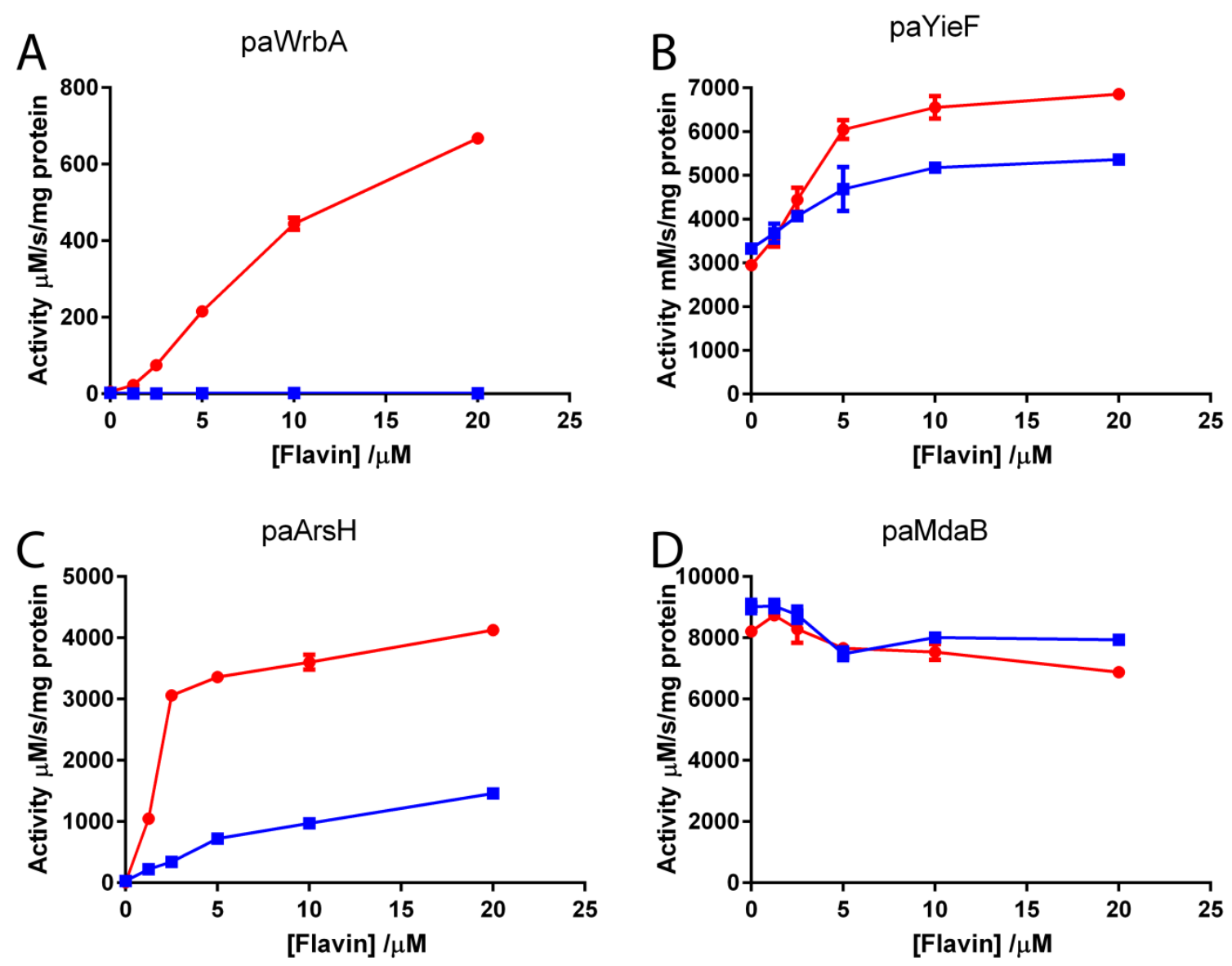

Figure 6

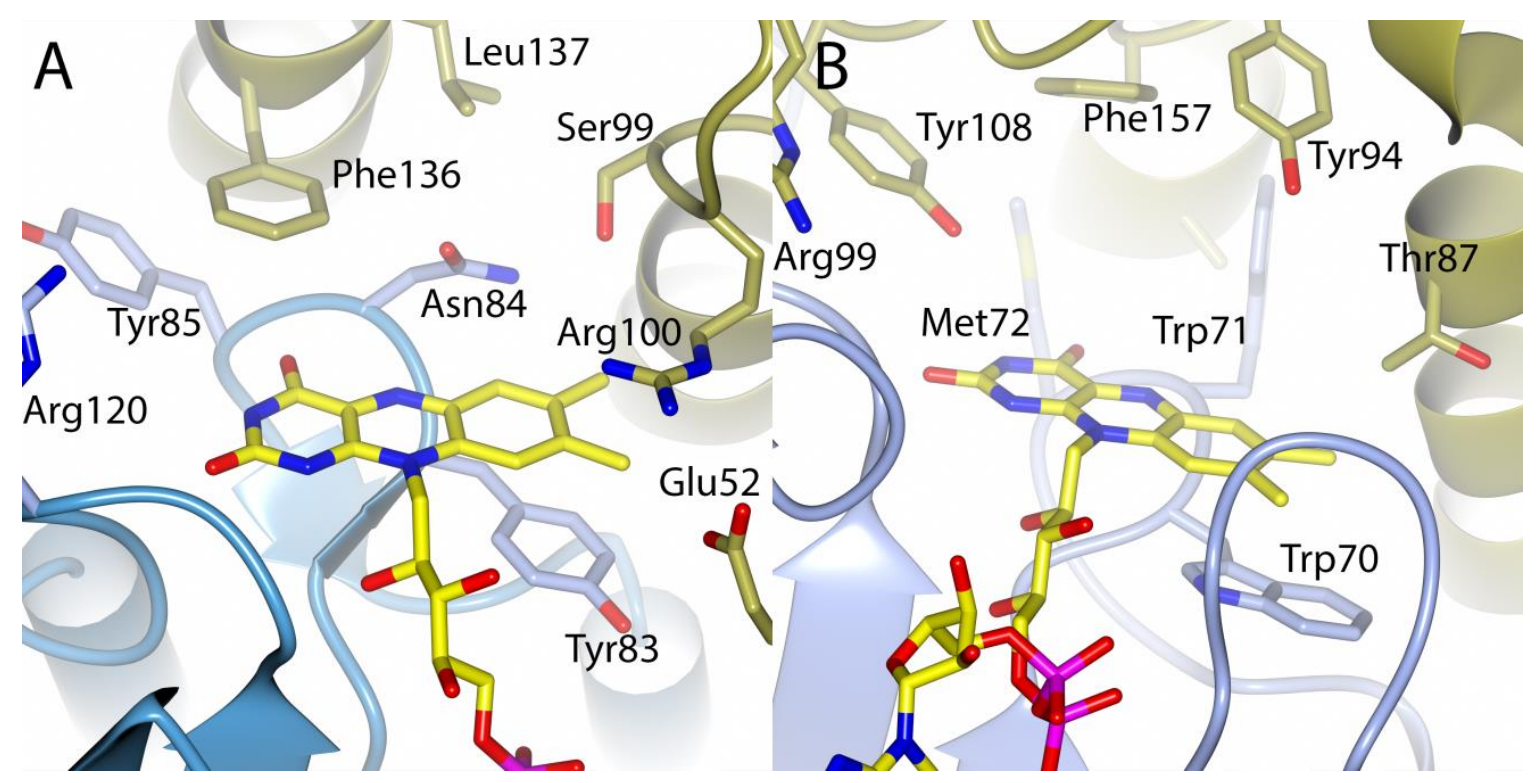

Figure 7 


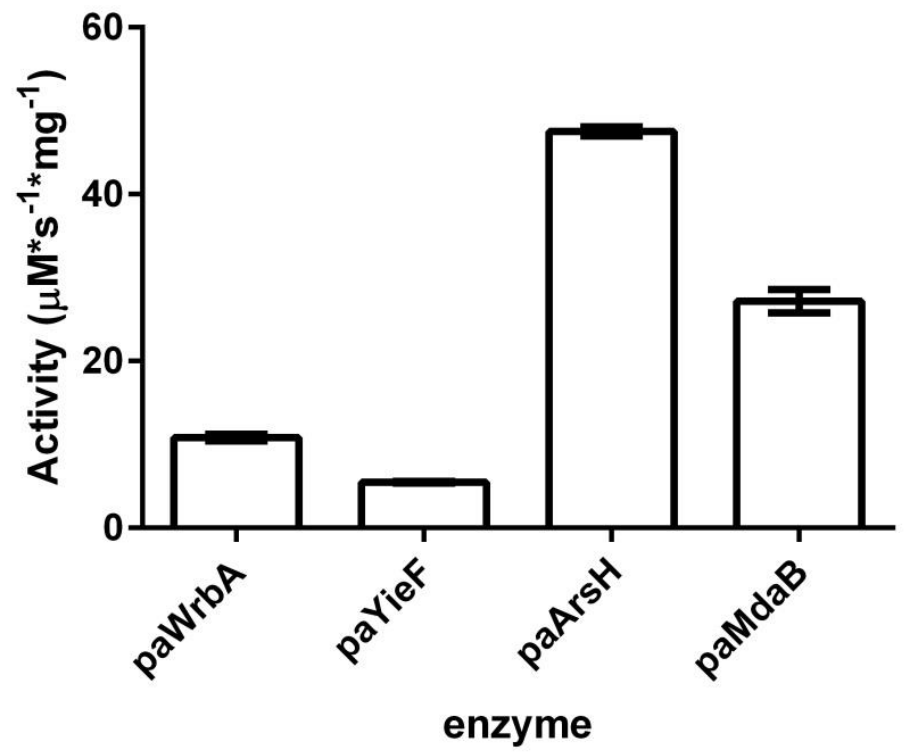

Figure 8 